\title{
Low-Density Ordered Phase in Brownian Dipolar Colloidal Suspensions
}

\author{
Amit K. Agarwal* and Anand Yethiraj ${ }^{\dagger}$ \\ Department of Physics and Physical Oceanography, Memorial University, St. John's, Newfoundland and Labrador, AlB 3X7, Canada
} (Received 27 October 2008; revised manuscript received 11 December 2008; published 11 May 2009)

\begin{abstract}
We study the low volume fraction and electric field phase behavior of a Brownian colloidal suspension. On the application of a uniform ac field, we find a novel phase where chains of particles aggregate to form a well defined cellular network, consisting of particle-free "voids" surrounded by a percolating network of particle-rich walls. This cellular structure is stable to very long times, indicative of an equilibrium thermodynamic phase. The cell-cell spacing is not sensitive to the concentration of the sample but scales with sample thickness. Any self-consistent mechanism for the existence of this void phase must consist of long-ranged repulsions and shorter-ranged attractions.
\end{abstract}

DOI: 10.1103/PhysRevLett.102.198301

PACS numbers: 47.57.J-, 64.70.pv

Brownian colloidal suspensions with tunable interactions are important model systems for understanding phase transitions in atomic systems [1-4]. Real-space microscopy and light scattering experiments have been carried out on crystal nucleation dynamics $[5,6]$ as well as structure and dynamics at the colloidal glass transition [7-9]. In the presence of an external electric field, Brownian colloidal spheres experience an anisotropic dipolar interaction and form chains (the "string fluid") and multichain aggregates [10,11], as well as body centered tetragonal (BCT) crystals [12-14]. Multiple competing interactions lead to richer phase behavior. In colloid-polymer mixtures both network-forming gel phases and glassy behavior can be observed by control of competing attractive and repulsive interactions $[15,16]$. Anisotropic interactions between colloids [17] in a liquid crystal medium also lead to a network of particle aggregates [18]. In dipolar colloids with added electrostatic repulsions, numerous crystalline phases are observed $[19,20]$. The dipolar interaction is of great interest because it consists of both competing on-axis (along the electric field) attractions and in-plane repulsions, and because it is an important driving force for nanoparticle selforganization [21].

Simulations of spheres with both dipolar and van der Waals interactions have shown many variants (linear aggregates, droplets, columns) of phase separation into regions of higher and lower densities [22,23]. However, structure formation in the low-density regime of Brownian colloidal suspensions in an electric field has not attracted much experimental attention. Recently, interesting field induced cellular structures were reported in a granular medium [24], but the mechanism (and whether they represented equilibrium structures) was not clear.

In this Letter, we report real-space confocal microscopy studies of the effect of an ac external electric field on the low-density structure of Brownian colloidal suspensions. We report the existence of a novel "gel-like" phase at low volume fractions $(\phi<4.0 \%)$ where chains of particles aggregate in time to form a cellular structure composed of "voids" - particle-free domains enclosed completely by particle-rich walls. We propose that the competition between the shorter-ranged attraction and the longer-ranged repulsion between chains could be responsible for this field-reversible cellular structure. This opens up new possibilities for patterned materials: unlike the depletion interaction, which is controlled by the addition of polymers, the chain-chain interaction is externally switchable.

Experiments were conducted with fluorescent-labeled core-shell silica microspheres (diameter $2 a=0.8 \mu \mathrm{m}$, polydispersity $\approx 3.8 \%$, synthesized as in [25]) suspended in a refractive index matched solvent of water:dimethyl sulfoxide (15:85 by volume), with a measured Debye screening length $\kappa^{-1}=50 \mathrm{~nm}$ [26] $(\kappa a=8)$. The volume fraction $\phi$ of suspension was varied from $0.07 \%$ to $12.0 \%$. The suspension was contained between two transparent, conducting (indium tin oxide glass) electrodes and imaged using a Nikon $\mathrm{C} 1$ confocal microscope. Image analysis was carried out in the application IDL [26].

Upon application of an electric field (1 MHz, ac), particles form strings along the field direction almost instantly.

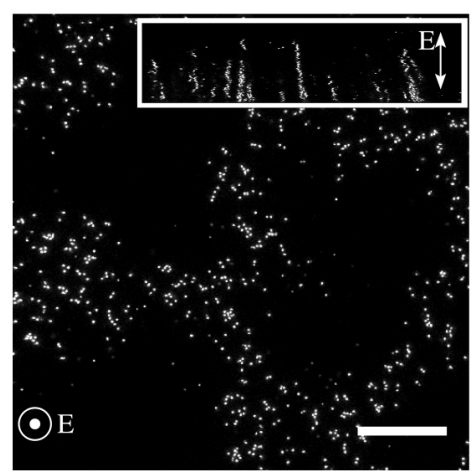

FIG. 1. Confocal micrograph ( $x-y$ cut) at an external field $E_{0 P}=1.0 \mathrm{~V} / \mu \mathrm{m} \quad(18 \mu \mathrm{m}$ above bottom plate, $\phi=0.6 \%$, 100× objective, scale bar $25 \mu \mathrm{m}$ ). Inset: $y$ - $z$ cut of sample (thickness $\approx 60 \mu \mathrm{m}$, width $\approx 125 \mu \mathrm{m}$ ) shows chains along $E_{0 P}$. 


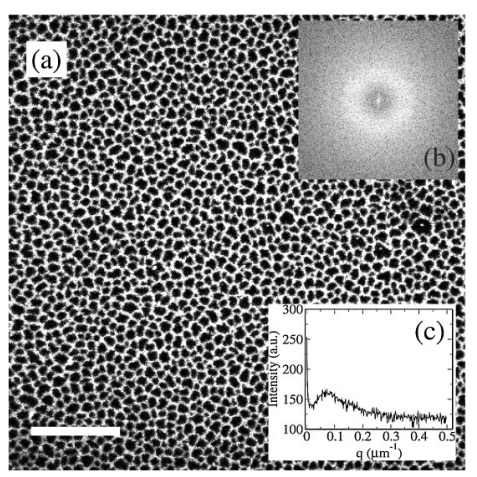

FIG. 2. (a) A well developed cellular structure in Brownian colloids (sample at $\phi=0.6 \%, 4 \times$ objective, scale bar $625 \mu \mathrm{m}$ ) at $E_{0 P}=1.0 \mathrm{~V} / \mu \mathrm{m}$. (b) A 2D Fourier transform of (a). (c) The peak in the average radial intensity profile in (b) corresponds to the center-center distance $d_{C C}$ between voids.

We observe string-fluid-BCT coexistence for $\phi>4 \%$ (consistent with [14,20]). At low volume fractions $(0.07 \%<\phi<4.0 \%)$, a cellular interconnecting particulate network with isolated particle-free voids appears about 2 minutes after the field is turned on. A confocal microscope image for $\phi=0.6 \%$ at a zero-to-peak value of electric field $E_{0 P}=1.0 \mathrm{~V} / \mu \mathrm{m}$ is shown in Fig. 1 (the $x-y$ plane shown is perpendicular to the applied electric field, along $z$ ). The inset of Fig. 1, a two-dimensional (2D) slice in the $y-z$ plane, shows that each feature in the $x-y$ plane corresponds to a chain along $z$ [27]. Because of the gravitational field, even though chains do not necessarily nucleate at the bottom plate, they are attached to the bottom plate in steady state. The average length of these chains increases with the applied electric field. To improve statistics (the voids' dimensions are $\approx 50 \mu \mathrm{m}$ ) we quantify the cellular structure using low magnification $(4 \times$ and $10 \times$ ) images. A $4 \times$ image of a well developed cellular network [Fig. 2(a)] shows particle-rich domains (as white spots) enclosing particle-free domains (or voids). Such cellular structures were recently reported in granular materials at low concentrations [24]; the authors conjectured that the absence of gravity in their system could somehow be implicated. Previous work on Brownian dipolar colloids (on samples with $\phi>10 \%$ [14]) had not observed these structures. The 2D Fourier transform of the image in Fig. 2(b) shows an isotropic ring which indicates the absence of in-plane anisotropy in the cellular structure. The peak (at $q=0.07 \mu \mathrm{m}^{-1}$ ) of the average radial intensity profile in Fig. 2(c) corresponds to the mean center-tocenter distance $\left(d_{C C}\right)$ between neighboring voids.

Figure 3 shows a series of images acquired as a function of applied electric field (zero-to-peak value $E_{0 P}$ ) for $\phi=$ $0.6 \%$. We waited at least 30 minutes before taking the first image and 10 minutes after each field increment to ensure a stationary structure. Voids first appeared [Fig. 3(c)] at $E_{0 P} \approx 0.66 \mathrm{~V} / \mu \mathrm{m}$. The distance between two neighboring voids $\left(d_{C C}\right)$ as a function of applied electric field [Fig. 3(i)] for three different sample concentrations (error bars reflect a single pixel in the Fourier transformed image; the sample thickness in these samples was $37.5 \pm 2.5 \mu \mathrm{m}$ ) appears to show no systematic dependence. The solid line represents the average of all the values shown in Fig. 3(i).

Next we determine the characteristic size of the voids. To this end, we divide images $(512 \times 512$ pixels $)$ into $N \times$ $N$ small boxes ( $N$ is an integer less than 128) and plot a histogram of the average gray level of boxes for different box sizes. For an image with no cellular structure [Fig. 3(a)], the histogram is a single distribution at all box sizes [Fig. 4(a)] with the peak of the distribution being close to the mean gray level. For a developed cellular structure [Fig. 3(h)], there is a threshold number of boxes $[10 \times$ 10 in this case, see Fig. 4(b)] above which the highest frequency is at zero gray level. This threshold is our estimate of the characteristic void size. In Fig. 4(c), we show the variation of void size as a function of applied electric field. While the center-to-center distance between two neighboring voids [see Fig. 3(i)] is insensitive to the applied field, the characteristic void size increases.

We probe the long-time dynamics of the voids next. In Fig. $5\left(\phi=0.15 \%, E_{0 P}=0.65 \mathrm{~V} / \mu \mathrm{m}\right)$ the time evolution of the void size is tracked from seconds to hours (see supplementary movies [27]). We fit the data to an exponential of the form $A_{\infty}\left(1-\exp \left[\left(t_{0}-t\right) / \tau\right]\right)$, where $A_{\infty} \approx$ $63 \mu \mathrm{m}$ (signifying the fully developed void size), $t_{0} \approx$ $148 \mathrm{sec}$ (signifying the time for the onset of the voids),

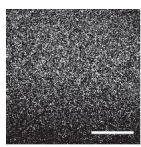

(a)

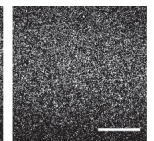

(b)

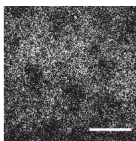

(c)

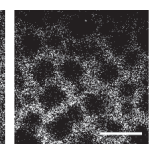

(d)

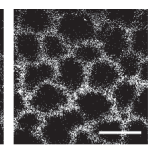

(e)

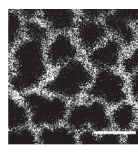

(f)

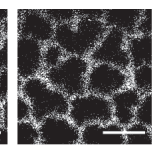

(g)

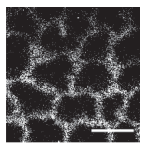

(h)

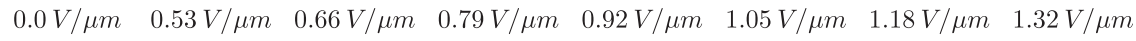

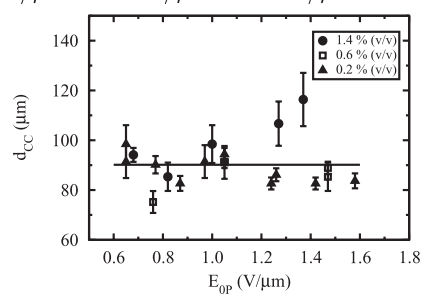

(i)
FIG. 3. (a)-(h) Formation of voids with increasing electric field $E_{0 P}$ (images of sample at $\phi=0.6 \%, 40 \times$ objective, scale bar $100 \mu \mathrm{m}$ ). (i) Fourier transform of low magnification images (samples at $\phi=0.2 \%, 0.6 \%$ and $1.4 \%$, $4 \times$ and $10 \times$ objectives) yields the distance $\left(d_{C C}\right)$ between two neighboring voids. This distance is insensitive to field strength for a given sample thickness (37.5 $\pm 2.5 \mu \mathrm{m}$ for these experiments). 


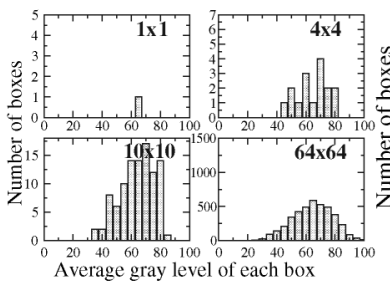

(a) $0 \mathrm{~V} / \mu \mathrm{m}$

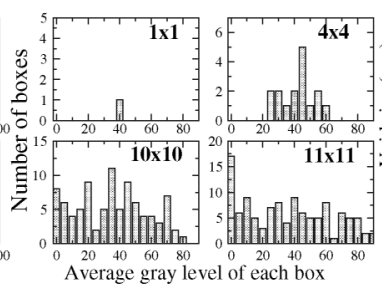

(b) $1.32 \mathrm{~V} / \mu \mathrm{m}$

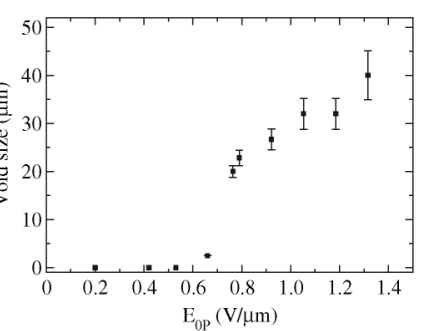

(c)

FIG. 4. Box analysis to obtain void size. Each image $(512 \times 512$ pixels $)$ is divided into $N \times N$ boxes where $N<128$ and is an integer. The average gray level of the boxes is then histogrammed. (a) Histogram for image in Fig. 3(a) shows a single-peaked distribution for all $N$ (peak located at average gray level of image). (b) Histogram for image in Fig. 3(h) exhibits a crossover from a single peak (at average gray level) at small $N$, to a distribution with a peak at zero gray level. The box size at this threshold is the characteristic void size. (c) Void size increases as a function of applied electric field.

and $\tau \approx 355 \mathrm{sec}$ (the time constant for void formation). Thus, the voids begin to appear $\approx 2.5$ minutes after applying the electric field. Observations up to 3.5 hours find no change in the cellular network. Given that the Brownian time scale of these colloids is $\approx 1$ second and the time scale for the onset of a fully developed cellular structure is $\approx 6$ minutes, the hours-long stationary state strongly suggests that the cellular structures containing voids are an equilibrium phase. The sharp increase from zero of the characteristic void size with applied field in Fig. 4(c) thus signifies a phase transition from string fluid to the void phase.

The void phase is qualitatively analogous (although richer, being field tunable and reversible) to networkforming structures in colloid-polymer systems [15,28], which also have competing interactions. The presence of a peak in the structure factor at length scales that are 5 times the nearest neighbor spacing has been observed in simulations involving short-range attractions and longrange repulsions [29]. This peak has been associated with the distance between neighboring clusters which are also observed in experiments. The length scale of our characteristic structures is however on the order of 50 times the

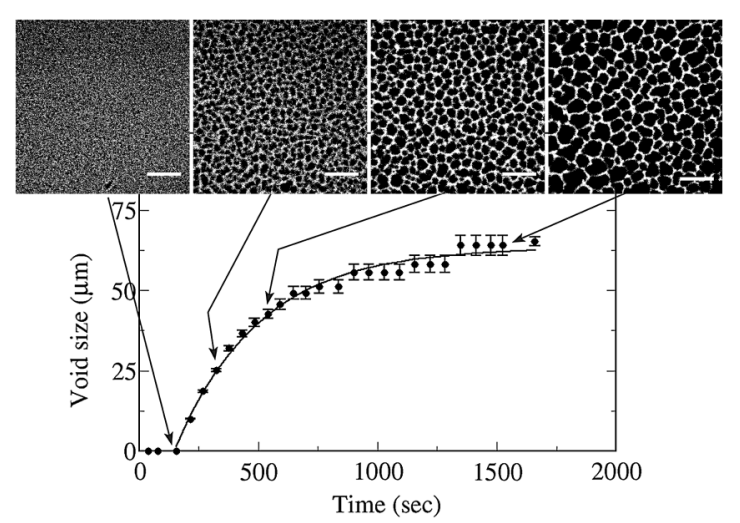

FIG. 5. Four snapshots in the long-time evolution of voids $\left(\phi=0.15 \%, E_{0 P}=0.65 \mathrm{~V} / \mu \mathrm{m}, 10 \times\right.$ objective, scale bar $250 \mu \mathrm{m}$, sample thickness $100 \mu \mathrm{m}$ ). The characteristic void size, calculated by the box analysis, increases with time, and is well fit (solid line) to a saturating exponential. nearest neighbor spacing. To rationalize this observation, we consider the interactions between two chains of spheres along the direction of the applied field [Fig. 6(a), inset]. As is known [12], dipolar spheres repel each other when $\theta>$ $54.7^{\circ}(\theta$ is the angle between the electric field and the line joining their centers), and attract otherwise. For chains, the interaction is a sum of the sphere-sphere interactions for each pair of spheres (one in each chain). At very short distances perpendicular to the chain, the chain-chain interaction is attractive. The range of the attractions depends on the importance of chain fluctuations [30] as well as the presence of other (e.g., van der Waals) interactions. When the minimum angle $\theta_{\min }$ satisfies $\theta_{\min }>54.7^{\circ}$ (corresponding to chain separations $d>L$ ), dipolar chains will certainly repel each other. We can test chain length dependence experimentally: Fig. 6(a) shows that $d_{C C}$ (each point is an averaged value of 2 to 20 data points) increases with sample thickness. Furthermore, simplistically assuming single-particle-thick network walls at a distance $d \approx L$ apart, we calculate an upper threshold of $\phi \approx 1 \%$. This is self-consistent with the experimental concentrations $(\phi<4 \%)$ at which voids are seen.
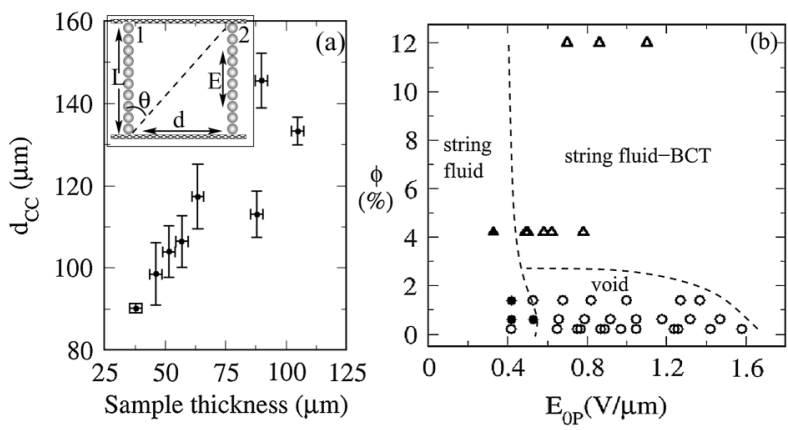

FIG. 6. (a) Inset: Two dipolar chains of length $L$ separated by distance $d$ always repel for $d>L$, but attract for much smaller separations. Body: The center-to-center distance $d_{C C}$ (error bars reflect single pixel in Fourier image) between voids increases with sample thickness. (b) The electric field and volume fraction phase diagram for $0.8 \mu \mathrm{m}$ silica particles (sample thickness, 35-45 $\mu \mathrm{m}$ ); empty circles, void phase; empty triangles, stringfluid-BCT coexistence; filled symbols, string fluid. 
In Fig. 6(b) we report the low volume fraction phase diagram (with suggestive phase boundaries) as a function of applied electric field. For $E_{0 P}<0.5 \mathrm{~V} / \mu \mathrm{m}$ there is a string-fluid phase for all volume fractions. The void phase exists for $\phi<4 \%$ and $E_{0 P}>0.5 \mathrm{~V} / \mu \mathrm{m}$. At $\phi>4 \%$ and $E_{0 P} \approx 0.6 \mathrm{~V} / \mu \mathrm{m}$ we confirm the coexistence of isolated chains and columnar clusters of BCT crystallites [27], as previously reported [14]. Experiments with bigger $(1.2 \mu \mathrm{m})$ silica particles also exhibited all the essential features of the void phase [27].

The phase diagram involves a percolation of the particulate network at large volume fractions, a depercolation to separated particulate clusters (with percolating particlefree domains, i.e., no voids) at $\phi \approx 10 \%$, and as we now find, an unexpected reentrant percolation at $\phi<4 \%$. While only string to BCT order has been observed previously for dipolar hard spheres $[13,14]$, other curious columnar phases (including a square array of columnar clusters [31]) have been predicted. The reentrant percolation of clusters is particularly unusual because the clusters are noncompact (see Fig. 1), with walls of thickness $\approx 10 \mu \mathrm{m}$. The wall thickness and the noncompactness together suggest a shallow attraction for $r<10 \mu \mathrm{m}$. Simulations of magnetic dipolar colloids in the presence of an external field and with an additional Lennard-Jones interaction $[22,23]$ produce structures that are most similar to those seen in our experiments. We conclude that added attractions make cluster formation easier. In particular, Richardi, Pileni, and Weis [23] have varied system thickness in the field (and chain length) direction, and observed both compact and noncompact columnar aggregates. Experimentally, the BCT clusters in the string-fluid-BCT region in Fig. 6(b) are composed of neighboring chains that are staggered by half a particle diameter (increasing dipolar attractions). In the void region of the phase diagram neighboring chains are not staggered, and correspondence with reported simulations [22,23] is better. Finally, it is likely that the local preference for high surface area noncompact structures is the cause of the observed reentrant percolating network at ultralow packing. Ultralow-density equilibrium cellular structures have potential applications as structured [32] macroporous materials. Typical lowdensity cellular materials are $>10 \%$ by weight solid [32]: this phase is $10-100$ times emptier.

This work was supported by the Natural Sciences and Engineering Research Council of Canada. We acknowledge numerous fruitful discussions with Ivan SaikaVoivod, and Ning Li for the synthesis of silica particles.

\footnotetext{
*amit@mun.ca

†ayethiraj@mun.ca
}

[1] P. N. Pusey and W. van Megen, Nature (London) 320, 340 (1986).

[2] A. P. Gast and W. B. Russel, Phys. Today 51, 24 (1998).

[3] A. Yethiraj, Soft Matter 3, 1099 (2007).

[4] E. B. Sirota et al., Phys. Rev. Lett. 62, 1524 (1989).

[5] P. Wette, H. J. Schope, and T. Palberg, J. Chem. Phys. 123, 174902 (2005).

[6] U. Gasser, E. R. Weeks, A. Schofield, P. N. Pusey, and D. A. Weitz, Science 292, 258 (2001).

[7] W. K. Kegel and A. van Blaaderen, Science 287, 290 (2000).

[8] E. R. Weeks, J. C. Crocker, A. C. Levitt, A. Schofield, and D. A. Weitz, Science 287, 627 (2000).

[9] S. R. Williams and W. van Megen, Phys. Rev. E 64, 041502 (2001).

[10] J. E. Martin, J. Odinek, T. C. Halsey, and R. Kamien, Phys. Rev. E 57, 756 (1998).

[11] M. Parthasarathy and D. J. Klingenberg, Mater. Sci. Eng., R 17, 57 (1996).

[12] T. C. Halsey and W. Toor, Phys. Rev. Lett. 65, 2820 (1990).

[13] R. Tao and J. M. Sun, Phys. Rev. Lett. 67, 398 (1991).

[14] U. Dassanayake, S. Fraden, and A. van Blaaderen, J. Chem. Phys. 112, 3851 (2000).

[15] K. N. Pham et al., Science 296, 104 (2002).

[16] A. M. Puertas, M. Fuchs, and M. E. Cates, Phys. Rev. Lett. 88, 098301 (2002).

[17] P. Poulin, H. Stark, T. C. Lubensky, and D. A. Weitz, Science 275, 1770 (1997).

[18] S. P. Meeker, W. C. K. Poon, J. Crain, and E. M. Terentjev, Phys. Rev. E 61, R6083 (2000).

[19] A. Yethiraj and A. van Blaaderen, Nature (London) 421, 513 (2003).

[20] A.-P. Hynninen and M. Dijkstra, Phys. Rev. E 72, 051402 (2005).

[21] Z. Tang, N. A. Kotov, and M. Giersig, Science 297, 237 (2002).

[22] M. J. Stevens and G. S. Grest, Phys. Rev. E 51, 5976 (1995).

[23] J. Richardi, M. P. Pileni, and J.-J. Weis, Phys. Rev. E 77, 061510 (2008).

[24] A. Kumar, B. Khusid, Z. Qiu, and A. Acrivos, Phys. Rev. Lett. 95, 258301 (2005).

[25] A. van Blaaderen and A. Vrij, Langmuir 8, 2921 (1992).

[26] N. Li, M.Sc. thesis, Memorial University, 2008.

[27] See EPAPS Document No. E-PRLTAO-102-083921 for figure on BCT and cellular structures for $1.2 \mu \mathrm{m}$ particles, movies of time evolution, and a 3D data stack of the cellular structure. For more information on EPAPS, see http://www.aip.org/pubservs/epaps.html.

[28] P. J. Lu et al., Nature (London) 453, 499 (2008).

[29] F. Sciortino, S. Mossa, E. Zaccarelli, and P. Tartaglia, Phys. Rev. Lett. 93, 055701 (2004).

[30] T. C. Halsey and W. Toor, J. Stat. Phys. 61, 1257 (1990).

[31] J.-J. Weis and D. Levesque, J. Chem. Phys. 125, 034504 (2006).

[32] M. Wu, T. Fujiu, and G. L. Messing, J. Non-Cryst. Solids 121, 407 (1990). 